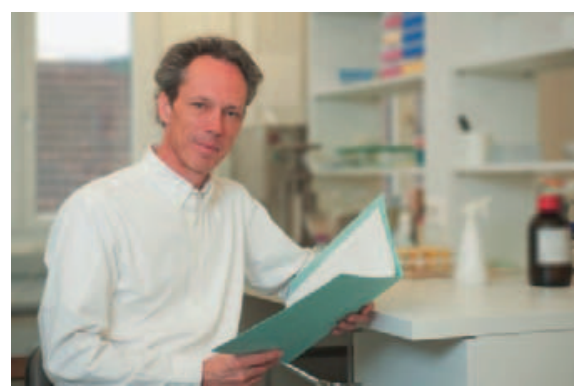

Sehr geehrter Herr Dr.

Baumgartner, 2017 ist es nun genau 100 Jahre her, dass die Ärztin Ita Wegman erstmals ein Mistelextrakt gezielt für die Behandlung von Krebspatienten eingesetzt hat. Was ist während dieser Zeit geschehen und wie wurde das anthroposophische Mistelpräparat weiterentwickelt?

Das 1917 von Ita Wegman eingesetzte Mistelpräparat Iscar wurde schon bald den Grundlinien der anthroposophischen Mistelpharmazie entsprechend optimiert und seither ständig weiterentwickelt. So werden durch die Gewinnung von Extrakten aus der Sommer- und der Wintermistel die saisonal unterschiedlichen Mistelwirkstoffe berücksichtigt und deren Qualitäten durch ein spezifisches Mischverfahren noch gesteigert. Dieser Maschinenprozess als zentraler Schritt in der Mistelpharmazie geht auf Angaben von Rudolf Steiner zurück, konnte aber erst 1972 dank Errungenschaften der Maschinenbauindustrie in den angestrebten physikalischen Rahmenbedingungen realisiert werden. Auch diese Bemühungen haben sich bewährt. So zeigten verschiedene Untersuchungen an Pflanzenkeimlingen, dass dieser spezifische Verarbeitungsprozess die Wirkung des Mistelpräparats signifikant zu steigern vermag. Heute sind die gesamte Herstellung wie auch die Qualitäts-

\title{
Die Mistel - faszinierende Pflanze mit spannendem Wirkpotenzial
}

Herr Dr. sc. nat. Stephan Baumgartner studierte Physik, Mathematik und Astronomie an der Universität Basel. Es folgten ein Forschungsaufenthalt an der Mathematisch-Astronomischen Sektion am Goetheanum, Dornach, ein Doktorat in Umweltnaturwissenschaften an der ETH Zürich sowie ein PostDoc in der Abteilung Umweltphysik der EAWAG (Eidgenössische Anstalt für Wasserversorgung, Abwasserreinigung und Gewässerschutz), Dübendorf. Seit 1996 ist er als Mitarbeiter in der Abteilung Grundlagenforschung im Institut Hiscia, Verein für Krebsforschung, Arlesheim, sowie am Institut für Komplementärmedizin (IKOM) der Universität Bern zu je 50\% tätig. Am IKOM baute er die Abteilung Grundlagenforschung Anthroposophische Medizin und Homöopathie mit auf. Seit 2009 ist Dr. Baumgartner als Dozent an der Universität Bern aktiv. Seit 2011 ist er zudem wissenschaftlicher Mitarbeiter an der Fakultät für Gesundheit der Universität Witten-Herdecke, wo er 2013 in experimenteller Medizin habilitierte.

kontrolle auf dem neuesten Stand der Technik, sprich GMP(«Good Manufacturing Practice»)-konform.

Ein nicht zu unterschätzender Entwicklungsfaktor ist zudem die Kultivierung der Mistel. Lange Zeit ging man davon aus, dass die Mistel auf der Eiche nicht zu kultivieren sei. Durch intensive Forschungen liessen sich Mitte der 1970er-Jahre jedoch praktikable Methoden entwickeln. Inzwischen ist durch den nachhaltigen Aufbau betriebseigener Mistelbestände die Versorgungssicherheit nicht nur für die seltene Eichenmistel, sondern auch die stark gefährdete Ulmenmistel gewährleistet.

Ebenfalls zu erwähnen sind die globale Verbreitung und die Anerkennung der Misteltherapie. Aufgrund der Beobachtungen und Erfahrungen von Ita Wegman mit dem Mistelpräparat Iscar fand bereits 1920 eine erste Schulung für anthroposophische Ärzte statt. Die Misteltherapie bei Krebs ist inzwischen weltweit verbreitet und im deutschsprachigen Raum bis heute die wichtigste komplementärmedizinische Behandlung in der Onkologie.

\section{Wie ist die ISCADOR AG entstanden und welche Rolle spielen hierbei der Verein für Krebsforschung (VfK) und das Institut Hiscia?}

1935 gründeten die Ärzte Ita Wegmann und Werner Kaelin sowie Rudolf Hauschka und Lina Kaelin den VfK. Dieser sollte vor allem die Entwicklung des Mistelpräparats ISCADOR, das 1926 bei den Behörden angemeldet worden war, weiter vorantreiben und die Misteltherapie bei Krebs weiter ausarbeiten.

Der Arzt Alexander Leroi gründete dann 1949 aus dem VfK heraus das Forschungsinstitut Hiscia, in dem die Weiterentwicklung des Maschinenprozesses, die Mistelkultivierung sowie die pharmazeutische Optimierung von Mistelextrakten und Wirkstoffen im Mittelpunkt stehen. 
2014 wurde die Iscador AG aus dem VfK als eigenständiges pharmazeutisches Unternehmen ausgegliedert. Die Iscador AG übernahm die Herstellung wie auch den weltweiten Vertrieb des Mistelpräparats ISCADOR.

\section{Inwiefern können Mistelpräparate in der integrativen Onkologie oder auch in anderen Bereichen der Komplementärmedizin eingesetzt werden?}

Die Misteltherapie übernimmt eine wichtige Rolle in der integrativen Onkologie. Viele Betroffene fragen nach komplementären Therapiemöglichkeiten und möchten aktiv etwas für sich tun. Die Misteltherapie ist das mit Abstand am besten erforschte komplementäre Therapieverfahren in der Onkologie und nachweislich wirksam. Adjuvant zu klassischen schulmedizinischen Methoden eingesetzt, kann sie deren Nebenwirkungen mindern und so die Compliance und die Lebensqualität der Patientinnen und Patienten verbessern [1, 2]. Studien zeigen, dass die Wirkungen von schulmedizinischen Standardtherapien durch eine Misteltherapie nicht beeinträchtigt werden $[3,4]$.

Zusätzlich können durch den Einsatz einer Misteltherapie auch Begleitbeschwerden einer Krebserkrankung wie beispielsweise die bekannte tumorbedingte Müdigkeit (Fatigue) sowie Schmerzen, Appetit- und damit einhergehend Gewichtsverlust gelindert werden [1].

\section{Welche direkten \\ Anwendungsbereiche in der onkologischen oder auch gynäkologischen Praxis bestehen Ihrer Erfahrung nach für die Mistelpräparate?}

Mistelpräparate können als subkutane Injektionstherapie bei allen soliden Tumoren zum Einsatz kommen. Je früher im Therapieverlauf begon- nen wird, desto besser. Je nach Tumorentität kommt die Mistel eines bestimmten Wirtsbaumes zum Einsatz. Es wird mit der niedrigsten Dosis begonnen und langsam hochdosiert, bis die Patientin oder der Patient die optimale Reaktion zeigt. Dabei kann die bzw. der Betroffene die Injektionen in der Regel selbst durchführen. Eine enge Begleitung in der Einstiegsphase ist jedoch wichtig. Wie bereits erwähnt, kann die Misteltherapie zusätzlich zu klassischen Therapien wie Chemo- und Strahlentherapie eingesetzt werden. Nach Abschluss dieser Standardtherapien empfiehlt sich jedoch eine Fortsetzung der Misteltherapie, da sie das Rezidiv-Risiko zu senken vermag [5].

\section{Welche weitere Forschung ist vonnöten, um das Wirkspektrum der Mistel noch besser kennen zu lernen bzw. den Einsatzbereich zu erweitern?}

Um die Wirksamkeit weiter zu verbessern, sind Forschungen insbesondere in zwei Bereichen im Gange. So wird untersucht, ob hinsichtlich der Anwendungsweise Optimierungspotenzial besteht - also wie z.B. Lokalisierung und Zeitpunkt der Applikation oder auch ISCADOR-Sorten von unterschiedlichen Mistelwirten sowie mögliche Kombinationen von Sorten die Wirksamkeit beeinflussen.

Des Weiteren wird daran geforscht, wie die Mistelpharmazie zugunsten einer Wirkungssteigerung optimiert werden kann. Dies betrifft zum einen die Extrakt-Herstellung, bei der weitere Inhaltstoffe verfügbar gemacht werden können. In diesem Zusammenhang von besonderem Interesse sind die lipophilen Inhaltsstoffe, auf deren Potenzial Rudolf Steiner bereits 1920 aufmerksam gemacht hat. Darüber hinaus sind auch das pharmazeutische Herstellungsverfahren und insbesondere der Maschinenprozess weiterhin Gegenstand der Forschung.
Was fasziniert Sie an der Mistel und deren Wirkpotenzial am meisten?

Einerseits ist die Mistel hinsichtlich ihrer Biologie eine äusserst faszinierende Pflanze. Sie wurzelt nicht im Erdboden, sondern benötigt für ihr Wachstum einen Wirtsbaum; sie wächst äusserst langsam, und zwar nicht in der Vertikalen, sondern in sich zentriert zu einem Kugelbusch. Im Vergleich zu anderen Blütenpflanzen zeigt sie ausserdem einen besonderen jahreszeitlichen Rhythmus. So blüht sie ausgangs des Winters und benötigt anschliessend etwa 9 Monate, bis ihre Früchte im Spätherbst zur Reife kommen.

Ebenso spannend ist jedoch ihr Wirkpotenzial: So ist ihre Wirkung nicht auf den Tumor begrenzt, sondern erstreckt sich auf den Menschen als Ganzes. Direkten Einfluss auf das Tumorwachstum nimmt sie durch ihre zytotoxischen und immunmodulierenden Eigenschaften. Genauso beeindruckend und für die Betroffenen oftmals sehr wichtig sind aber auch ihre positiven Wirkungen auf Begleiterscheinungen der Krebskrankheit wie Appetitlosigkeit, Schmerzen, depressive Verstimmung und die Selbstregulation. Hier decken sich die Ergebnisse der Studien von Grossarth-Maticek mit den frühen Beobachtungen von Ita Wegmann: Die Patienten sind wieder mehr bei sich und ergreifen neue Initiativen zur Gestaltung ihres Lebens [6]. Dies macht die Misteltherapie zu einer wertvollen Behandlung in der integrativen Krebstherapie. Sie gibt dem Leben mehr Qualität.

\section{Herzlichen Dank für das Interview!}

Interview: Alexander Eitner 


\section{Literatur}

1 Tröger W, et al: Quality of life of patients with advanced pancreatic cancer during treatment with mistletoe: a randomized controlled trial. Dtsch Arztebl Int 2014;111:493-502.

2 Tröger W, et al: Quality of life and neutropenia in patients with early stage breast cancer: a randomized pilot study comparing additional treatment with mistletoe extract to chemotherapy alone. Breast Cancer (Auckl) 2009;3:3545.
3 Tröger W, et al: Five-year follow-up of patients with early stage breast cancer after a randomized study comparing additional treatment with Viscum album (L.) extract to chemotherapy alone. Breast Cancer (Auckl) 2012; $1: 173-180$.

4 Weissenstein $U$, et al: Interaction of standardized mistletoe (Viscum album) extracts with chemotherapeutic drugs regarding cytostatic and cytotoxic effects in vitro. BMC Complement Altern Med 2014;14:6.
5 Grossarth-Maticek R, Ziegler R: Prospective controlled cohort studies on long-term therapy of breast cancer patients with a mistletoe preparation (Iscador). Forsch Komplementmed 2006;13:285-292.

6 Grossarth-Maticek R, et al: Synergieeffekte von Selbstregulation und Misteltherapie (Iscador) auf die Überlebenszeit bei Krebspatienten. Schweiz Zschr Ganzheitsmedizin 2004; 16 : 81-89. 